INPLASY

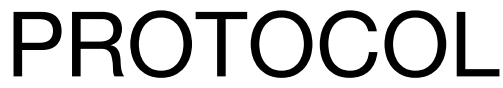

To cite: Chen et al.

Effectiveness of Chaihu-

Shugan-San in treatment of

Non-alcoholic Fatty Liver

Disease: A meta-analysis.

Inplasy protocol 2021100074.

doi:

10.37766/inplasy2021.10.0074

Received: 20 October 2021

Published: 20 October 2021

Corresponding author:

Hui Chen

chen_hui2018@163.com

Author Affiliation:

Guangdong Pharmaceutical

University; The First Affiliated

Hospital of Guangdong

Pharmaceutical University.

Support: None.

Review Stage at time of this submission: Preliminary

searches.

Conflicts of interest:

None declared.

\section{Effectiveness of Chaihu-Shugan-San in treatment of Non-alcoholic Fatty Liver Disease: A meta-analysis}

Chen, $\mathrm{H}^{1}$; Liu, X2 Meng, Z3; Huang, X4; Piao, S5; Guo, J6.

Review question / Objective: This study is to systematically evaluate the efficacy and safety of Chaihu-Shugan-San combined with or without conventional treatment for nonalcoholic fatty liver disease (NAFLD) was evaluated by metaanalysis.

Condition being studied: Non-alcoholic fatty liver disease (NAFLD) has become the most important chronic liver disease, affecting approximately 1.7 billion people worldwide, accounting for approximately $25 \%$ of the adult population. Studies have shown that it is closely associated with extrahepatic tumors, diabetes, cardiovascular disease, and metabolic syndrome. Finding an effective and safe interventional therapy is urgent because there is no specific treatment method yet. Recent studies have found that Traditional Chinese medicine has prominent advantages in the treatment of NAFLD.

INPLASY registration number: This protocol was registered with the International Platform of Registered Systematic Review and Meta-Analysis Protocols (INPLASY) on 20 October 2021 and was last updated on 20 October 2021 (registration number INPLASY2021100074).

\section{INTRODUCTION}

Review question / Objective: This study is to systematically evaluate the efficacy and safety of Chaihu-Shugan-San combined with or without conventional treatment for non-alcoholic fatty liver disease (NAFLD) was evaluated by meta-analysis.
Condition being studied: Non-alcoholic fatty liver disease (NAFLD) has become the most important chronic liver disease, affecting approximately 1.7 billion people worldwide, accounting for approximately $25 \%$ of the adult population. Studies have shown that it is closely associated with extrahepatic tumors, diabetes, cardiovascular disease, and metabolic 
syndrome. Finding an effective and safe interventional therapy is urgent because there is no specific treatment method yet. Recent studies have found that Traditional Chinese medicine has prominent advantages in the treatment of NAFLD.

\section{METHODS}

Search strategy: We will conduct literature searches from the following electronic databases: PUBMED, Web of Science, Cochrane Library, Embase, SinoMed, China National Knowledge Infrastructure (CNKI), China Science and Technology Journal Database, and Wanfang Database. The search strategy will include medical subject terms (MeSH) and keywords related to the treatment of NAFLD, and the search time will be set to the time of establishment of the database until October 2021. And by cross-checking the reference lists of all the included main studies and the list of related systematic reviews. Grey literature, Chinese Clinical Trial Registry, and Clinical Trials will also be searched as supplements.

Participant or population: NAFLD patients diagnosed in accordance with the guidelines will be included, without limitation of age, gender and race.

Intervention: Chaihu-Shugan-San combined with or without conventional treatment.

Comparator: Placebo or Conventional treatment.

Study designs to be included: Randomized controlled trials (RCTs) will be included.

Eligibility criteria: (1)NAFLD patients diagnosed in accordance with the guidelines will be included, without limitation of age, gender and race; (2)Interventions: The experimental group was administered Chaihu-Shugan-San combined with or without conventional treatment. (3) Comparison: the control group was treated with Placebo or Conventional treatment.; (4)Study design: randomized controlled trials will be included; (5) Outcome indicators: total effective rate, main blood lipid indexes (TC, TG), main liver function indexes (ALT, AST).

Information sources: The two researchers independently searched and extracted pieces of literature from PUBMED, Web of Science, Cochrane Library, Embase, SinoMed, China National Knowledge Infrastructure (CNKI), China Science and Technology Journal Database, and Wanfang Database after selecting keywords and matching free words. The search period is from the database establishment to October 2021, regardless of language. The search strategy will take the form of medical subject headings (MeSH) and keywords and optimize the search strategy according to the characteristics of different databases. Keywords include: "Chaihu-Shugan-San", "non-alcoholic fatty liver disease","nonalcoholic steatohepatitis", "randomized controlled trial", "clinical efficacy", etc.

Main outcome(s): The main outcomes will include total effective rate, main blood lipid indexes (TC, TG), main liver function indexes (ALT, AST).

Additional outcome(s): Additional outcomes will include but are not limited to clinical syndrome integral, Body Mass Index(BMI), insulin levels.

Data management: Two authors independently conducted research selection and data extraction. Any disagreements will be resolved through discussion until a consensus is reached or a third author is consulted. The extracted information includes several aspects: the overall characteristics of the study, the general characteristics of the subjects, the detailed information of the methodological information, the detailed information of the treatment plan, the detailed information of the control intervention measures (including conventional treatment drugs), the research results (including clinical and Laboratory parameters) and adverse effects. 
Quality assessment / Risk of bias analysis: The risk of bias of the included articles is based on the RCT bias risk assessment criteria provided by the Cochrane Collaborative Group. Random sequence generation, allocation hiding, blinding of participants and personnel, blinding of outcome evaluation, incomplete and selective outcome data are made by two researchers independently Report for evaluation, discrepancies will be resolved through discussion or referral to a third examiner.

Strategy of data synthesis: The results of the included studies will be descriptively synthesized, including participant characteristics and intervention details. We will calculate the effect size by weighted average/standard average deviation (for continuous results) and $95 \% \mathrm{Cl}$ odds ratio (OR) and $95 \% \mathrm{Cl}$ (for binary results). ChiSquared test and 12 test were used to test the heterogeneity of the included literature. When $P>0.1$ and $12<50 \%$, it indicates that there is no statistical heterogeneity between the studies; conversely, when P50 $\%$, it is considered that there is statistical heterogeneity between the studies. Use RevMan5.3 provided by the Cochrane Collaboration Network for meta-analysis. If there is clinical and methodological heterogeneity, a subgroup analysis will be performed to explore the source of the heterogeneity in the study.

Subgroup analysis: If sufficient trials are included, we will use subgroup analyses to explore potential sources of heterogeneity by age, duration of disease and interventions, or other factors that need to be considered.

Sensitivity analysis: We plan to conduct sensitivity analysis by deleting individual trials one by one and repeating metaanalysis to explore the reasons for the heterogeneity between studies.

Language: English.

Country(ies) involved: China.
Keywords: Chaihu-Shugan-San; NonAlcoholic Fatty Liver Disease; Metaanalysis; randomized controlled trials.

Contributions of each author:

Author 1 - Hui Chen.

Author 2 - Xinyu Liu.

Author 3 - Zhenzhen Meng

Author 4 - Xiaoqiang Huang.

Author 5 - Shanghua Piao.

Author 6 - Jiao Guo. 